\title{
Optimization of ruthenium as a buffer layer for non-collinear antiferromagnetic $M_{3} X$
} films

S. Kurdi ${ }^{1,}{ }^{*}$, P. Zilske ${ }^{2}$, X.D. Xu ${ }^{3, \dagger}$, M. Frentrup ${ }^{1}$, M.E. Vickers ${ }^{1}$, Y. Sakuraba ${ }^{3}$, G. Reiss ${ }^{2}$, Z.H. Barber $^{1}$ and J.W. Koo ${ }^{2,+}$

${ }^{1}$ Department of Materials Science and Metallurgy, University of Cambridge, CB3 0FS Cambridge, United Kingdom.

${ }^{2}$ Center for Spinelectronic Materials and Devices, Bielefeld University, D-33501 Bielefeld, Germany

${ }^{3}$ National Institute for Materials Science (NIMS), 1-2-1 Sengen, Tsukuba 305-0047, Japan

$\dagger$ Present address: College of Materials Science and Engineering, Hunan University, Changsha 410082, China

*sk862@cam.ac.uk

†jkoo@physik.uni-bielefeld.de

\begin{abstract}
Two thin film deposition routes were studied for the growth of high quality single crystalline $\mathrm{Ru}(0001)$ epitaxial films on $c-\mathrm{Al}_{2} \mathrm{O}_{3}$ substrates using $\mathrm{RF}$-magnetron sputtering. Such films are very important as buffer layers for the deposition of epitaxial non-collinear antiferromagnetic $\mathrm{Mn}_{3} X$ films. The first route involved depositing $\mathrm{Ru}$ at $700{ }^{\circ} \mathrm{C}$, leading to a smooth $30 \mathrm{~nm}$ thick film. Although, high resolution X-ray diffraction (HRXRD) revealed twinned Ru film orientations, the in-situ post-annealing eliminated one orientation, leaving the film orientation aligned with the substrate, with no in-plane lattice rotation and a large lattice mismatch (13.6\%). The second route involved deposition of $\mathrm{Ru}$ at room temperature followed by in-situ post-annealing at $700{ }^{\circ} \mathrm{C}$. Transmission electron microscopy confirmed a very high quality of these films, free of crystal twinning, and a $30^{\circ}$ in-plane lattice rotation relative to the substrate, resulting in a small in-plane lattice mismatch of $-1.6 \%$. X-ray reflectivity demonstrated smooth surfaces for films down to $7 \mathrm{~nm}$ thickness. $30 \mathrm{~nm}$ thick high quality single-crystalline $\mathrm{Mn}_{3} \mathrm{Ga}$ and $\mathrm{Mn}_{3} \mathrm{Sn}$ films were grown on top of the Ru buffer deposited using the second route as a first step to realize $\mathrm{Mn}_{3} X$ films for antiferromagnetic spintronics applications.
\end{abstract}




\section{Introduction}

In the past decade, antiferromagnets have come to the forefront for spin-electronics (spintronics) research. Utilizing antiferromagnets as a main component for spintronic devices allows for the realization of denser and more robust data storage capabilities compared to ferromagnetic devices due to the intrinsic properties of antiferromagnetic order ${ }^{1}$. However, in general, the manipulation of their magnetic order requires very large magnetic fields limiting their implementation. Antiferromagnetic systems, like non-collinear $\varepsilon-\mathrm{Mn}_{3} X(X=\mathrm{Sn}, \mathrm{Ge}, \mathrm{Ga})$, have shown properties that promise to overcome this bottleneck. Their topologically non-trivial band structure exhibiting Weyl nodes ${ }^{2}$ and their geometrical frustrated spin structure leads to the anomalous Hall ${ }^{3}$ and Nernst ${ }^{4}$ effects in addition to the magneto-optical Kerr effect ${ }^{5}$ which are forbidden in usual antiferromagnets. This is of great importance as it could lead to future spintronic devices in which antiferromagnets play the major role. Many of the recent studies have been on bulk $\operatorname{Mn}_{3} X\{X=\mathrm{Sn}, \mathrm{Ge})^{3,6}$, whilst thin film investigations have focused on polycrystalline or textured films for exchange bias applications ${ }^{7-10}$. However, epitaxial $\mathrm{Mn}_{3} \mathrm{Sn}$ films deposited on $\mathrm{Ru}(5 \mathrm{~nm})$ buffered $\mathrm{Y}: \mathrm{ZrO}_{2}$ substrates were not continuous due to island formation $^{9}$, making it not possible to measure their transport properties. In other reports quasiepitaxial $\mathrm{Mn}_{3} \mathrm{Sn}$ (112̄0) and (0001)-oriented thin films deposited on $\mathrm{Al}_{2} \mathrm{O}_{3}(1 \overline{1} 02)$ and $\mathrm{MgO}$ (111) substrates, respectively ${ }^{11}$, and epitaxial $\mathrm{Mn}_{3} \mathrm{Sn}$ films grown on $\mathrm{Ru}$ (5 nm) buffered $\mathrm{MgO}(111)^{12}$ showed dissimilar magnetization properties to those of bulk samples, implying that there is scope for improvement in preparation of these films.

In order to exploit the properties of $\mathrm{Mn}_{3} X$ for device applications, high quality epitaxial thin films are required. Although, $\mathrm{Mn}_{3} X$ films can be grown on a single crystalline substrate sharing the same crystal structure, e.g., $c$-plane sapphire $\left(c-\mathrm{Al}_{2} \mathrm{O}_{3}\right)$, a large lattice mismatch between $\mathrm{Mn}_{3} X$ films and the $c-\mathrm{Al}_{2} \mathrm{O}_{3}$ substrate hinders the smooth epitaxial growth of $\mathrm{Mn}_{3} X$ thin films. This constraint can be removed if one utilizes an appropriate buffer layer to minimize the lattice mismatch. In this work, we study hexagonal $\mathrm{Ru}$ as a buffer layer for $\mathrm{Mn}_{3} X$ films. $\mathrm{Ru}$ has very good lattice matching with $\mathrm{Mn}_{3} X$ in the $a$-plane: $\sim 0.2 \%, 1.3 \%, 4.5 \%$ misfit for $\mathrm{Mn}_{3} \mathrm{Ga}, \mathrm{Mn}_{3} \mathrm{Ge}$ and $\mathrm{Mn}_{3} \mathrm{Sn}$ respectively, suggesting that a highly epitaxial $\mathrm{Ru}$ buffer layer on $c-\mathrm{Al}_{2} \mathrm{O}_{3}$ will promote smooth epitaxial growth of $\mathrm{Mn}_{3} X$ films. In addition to the close lattice match, $\mathrm{Al}_{2} \mathrm{O}_{3}$ substrates are very stable, with no requirement for specific surface preparations. In contrast, $\mathrm{MgO}$ substrates require annealing to remove surface contamination ${ }^{13}$ and are known to lack consistency in quality ${ }^{14,15}$. 
We demonstrate that a $\mathrm{Ru}$ buffer layer on $c-\mathrm{Al}_{2} \mathrm{O}_{3}$ can effectively support the subsequent growth of highly epitaxial $\mathrm{Mn}_{3} X$ thin films. Although the growth of $\mathrm{Ru}$ on $c-\mathrm{Al}_{2} \mathrm{O}_{3}{ }^{16-20}$ has been reported and various techniques have been used to deposit thin $\mathrm{Ru}$ films, including pulsed laser deposition (PLD) ${ }^{18,19,21}$, e-beam evaporation ${ }^{22}$, metal-organic chemical vapour deposition $(\mathrm{MOCVD})^{23,24}$, and atomic layer deposition (ALD) $)^{25}$, there is some discrepancy between studies of the resultant crystalline orientation. In this work, we utilize RF magnetron sputtering $17,20,26,27$ to devise a thin film deposition procedure for high quality Ru thin films and demonstrate that a single-crystalline epitaxial $\mathrm{Ru}$ thin film is suitable for the subsequent growth of highly epitaxial $\mathrm{Mn}_{3} X$ thin films.

\section{Experimental Methods}

$\mathrm{Ru}$ thin films were deposited onto $c-\mathrm{Al}_{2} \mathrm{O}_{3}$ substrates using RF-magnetron sputter deposition from a 2 -inch diameter $\mathrm{Ru}$ target $(99.95 \%$ purity), in a UHV sputtering chamber built by BESTEC GmbH (Berlin), with a base pressure below 1 x $10^{-8} \mathrm{~Pa}$. The substrates were loaded directly from the supplier's (CrysTec $\mathrm{GmbH}$ ) vacuum-sealed packaging, with no further surface preparation. Film deposition took place at an $\operatorname{Ar}(6 \mathrm{~N})$ gas pressure of $0.2 \mathrm{~Pa}$. The substrates $\left(10 \times 10 \mathrm{~mm}^{2}\right)$ were rotated at $10 \mathrm{RPM}$ to ensure uniform deposition across the surface. A radiative heater, positioned at the back of the sample holder, allowed control of the growth temperature.

Two routes were followed for $\mathrm{Ru}$ thin film growth:

(i) deposition at a relatively high substrate temperature, $T_{\mathrm{s}}=700^{\circ} \mathrm{C}$, followed by in situ postannealing (in vacuum) at the same temperature, and

(ii) deposition at room temperature, followed by in situ post-annealing (in vacuum) at $T_{\mathrm{s}}=$ $700{ }^{\circ} \mathrm{C}$ (heating rate $=15^{\circ} \mathrm{C} \mathrm{min}^{-1}$ )

Further samples were grown via route (ii), followed by the deposition of $30 \mathrm{~nm} \mathrm{Mn}_{3} \mathrm{Ga}$ $\left(\mathrm{Mn}_{3} \mathrm{Sn}\right)$ films at $600{ }^{\circ} \mathrm{C}\left(350^{\circ} \mathrm{C}\right)$ on top of the $7 \mathrm{~nm}$ thick Ru-buffer via DC-magnetron cosputter deposition using 2-inch Mn:Ga (50:50 at\%, 99.97\% purity) / Mn:Sn (65:35 at\%, 99.9\% purity) and an additional $\mathrm{Mn}$ target (99.9\% purity). The $\mathrm{Mn}_{3} \mathrm{Ga}\left(\mathrm{Mn}_{3} \mathrm{Sn}\right)$ films were capped with a $2 \mathrm{~nm}$ Ru layer at room temperature. The deposition rates of $\sim 1.8 \AA \mathrm{s}^{-1}(\mathrm{Ru}), \sim 1.3 \AA \mathrm{s}^{-}$ ${ }^{1}\left(\mathrm{Mn}_{3} \mathrm{Ga}\right)$ and $\sim 1.1 \AA \mathrm{s}^{-1}\left(\mathrm{Mn}_{3} \mathrm{Sn}\right)$, were first calibrated in situ, using a quartz crystal microbalance, and confirmed by X-ray Reflectivity (XRR) measurement. The actual compositions of the films were $\mathrm{Mn}_{72 \pm 3} \mathrm{Ga}_{28 \pm 1}$ and $\mathrm{Mn}_{75 \pm 1} \mathrm{Sn}_{25 \pm 4}$, as determined by X-ray 
fluorescence (XRF), which are within the stable compositional range of the $D 0_{19}$ phase of $\mathrm{Mn}_{3} \mathrm{Ga}^{28}$ and $\mathrm{Mn}_{3} \mathrm{Sn}^{29}$.

High-resolution X-ray Diffraction (HRXRD) was performed using a four-circle Panalytical Empyrean vertical $\theta / \theta$ diffractometer with a hybrid 2-bounce primary monochromator. A proportional counter was used for the $2 \theta / \theta$ and $\varphi$ scans and a 2D PIXCEL position sensitive detector (PSD) for collecting the reciprocal space maps (RSMs). A Philips X'Pert Pro MPD $\theta / \theta$ diffractometer was used to obtain the XRR scans. Cross-sectional lamellae were produced using focused ion-beam (FEI Helios G4 UX) lift-out technique. The cross section of a $7 \mathrm{~nm}$ thick $\mathrm{Ru}$ film prepared via route (ii) was investigated using a FEI Titan G2 80-200 scanning/transmission electron microscope (S/TEM) with probe corrector operated at $200 \mathrm{kV}$. The images were taken in both annular dark field (ADF) and high angle annular dark field (HAADF) modes. A condenser aperture of $10 \mu \mathrm{m}$ was used to collect the selected area electron diffraction (SAED) patterns.

\section{Results and Discussion}

Route (i): Fig. 1 shows XRD and XRR results for three Ru films of nominal $5 \mathrm{~nm}, 15 \mathrm{~nm}$ and $30 \mathrm{~nm}$, grown at $T_{\mathrm{s}}=700{ }^{\circ} \mathrm{C}$, with a subsequent 20 minute, $700{ }^{\circ} \mathrm{C}$ anneal. Fig.1(a) shows XRD $2 \theta / \theta$ scans around the (0002) and (0004) Ru reflections, revealing that no other phases, nor orientations, are present. The film peaks are also observed in the same position implying a constant out-of-plane lattice parameter for all three thicknesses. Clear satellite peaks (thickness fringes), illustrating very uniform film growth, are observed for the $30 \mathrm{~nm}$ thick film, but are much less prominent for the thinner films. These observations are supported by the XRR scans in Fig.1(b), revealing sharp Kiessig fringes over the entire $5^{\circ}$ range for the $30 \mathrm{~nm}$ thick film, in which the roughness is determined to be $\sim 5.8 \AA$ from the scan simulation. However, the Kiessig fringes are relatively weak for the thinner films, indicating that they have a rougher surface, as observed by atomic force microscopy (AFM) (Supplementary Note 1). This is consistent with the shift of the critical edge towards lower angles, indicating a lower average density close to the $\mathrm{Ru}$ surface compared to thick well crystalline $\mathrm{Ru}$ films. Although the oscillations in the XRR signal are relatively weak for the thinner films, which makes a simulation and fit of the signal difficult, the periodicity of the oscillations of the scans suggest thicknesses of $\sim 21 \mathrm{~nm}$ and $\sim 10 \mathrm{~nm}$ (expected to be $15 \mathrm{~nm}$ and $5 \mathrm{~nm}$ respectively from the sputtering rates, assuming a uniform thickness). These observations correspond with the vertical coherence length calculation using Scherrer's formula ${ }^{30}$ (Supplementary Note 2). 
$\varphi$-scans from the $\mathrm{Ru}(10 \overline{1} 1)$ and $\mathrm{Al}_{2} \mathrm{O}_{3}(11 \overline{2} 3)$ reflections of the $30 \mathrm{~nm}$ film are shown in Fig.2(a), giving information about the in-plane film orientation. The lattice constants for $\mathrm{Ru}$ and $\mathrm{Al}_{2} \mathrm{O}_{3}$ are $a_{R u}=2.706 \AA(c=4.281 \AA)^{31}$ and $a_{A l_{2} O_{3}}=4.763 \AA\left(c_{A l_{2} O_{3}}=12.991 \AA\right)$, respectively. Therefore, the in-plane lattice misfit for $c$-plane $\mathrm{Ru}$ growth, if the axes line up, is $13.6 \%$. However, a $30^{\circ}$ in-plane rotation of the $\mathrm{Ru}$ film lattice relative to $c-\mathrm{Al}_{2} \mathrm{O}_{3}$ leads to an in-plane lattice mismatch of only $-1.6 \%$, since $a_{A l_{2} O_{3}} \sim 2 a_{R u} \cos 30^{\circ}$. This low lattice misfit configuration, with a $30^{\circ}$ in-plane lattice rotation, would lead to 6-fold symmetry with $\mathrm{Ru}(10 \overline{1} 1)$ peaks coincident with the $\mathrm{Al}_{2} \mathrm{O}_{3}(11 \overline{2} 3)$ reflections. However, the 6 broad grey peaks of Fig. 2(a) (marked with grey arrows) indicate an unrotated lattice. The narrower (and less intense) reflections between these peaks could be $30^{\circ}$-rotated $\mathrm{Ru}$. However, since the $\mathrm{Al}_{2} \mathrm{O}_{3}(11 \overline{2} 3)$ and $\mathrm{Ru}(10 \overline{1} 1)$ reflections have very similar diffraction conditions (for $\mathrm{Ru}(10 \overline{1} 1), 2 \theta=43.960^{\circ}, \chi=61.27^{\circ}$; and for $\left.\mathrm{Al}_{2} \mathrm{O}_{3}(11 \overline{2} 3), 2 \theta=43.352^{\circ}, \chi=61.21^{\circ}\right)$, these intermediate reflections could be trace signals from the very strong $\mathrm{Al}_{2} \mathrm{O}_{3}(11 \overline{2} 3)$ peaks. This is mirrored in the $\mathrm{Al}_{2} \mathrm{O}_{3}(11 \overline{2} 3) \varphi$-scan (bottom of Fig.2(a)), for which the narrow, intense peaks stem from the single crystal substrate, and the less intense intermediate peaks are traces of the $\mathrm{Ru}(10 \overline{1} 1)$ reflections.

To gain further information on the in-plane orientation of this $\mathrm{Ru}$ film, $\chi$ was set at $61.20^{\circ}$ and $2 \theta / \theta$ scans were taken at $\varphi=0^{\circ}$ and $\varphi=30^{\circ}$ (Fig. 2(b)). The $\mathrm{Al}_{2} \mathrm{O}_{3}$ peak only appears at $\varphi=0^{\circ}$, whilst a $\mathrm{Ru}$ peak appears at both angles, indicating the presence of two, twin-related ${ }^{32}$ film orientations, and confirming that all 12 peaks in the top trace of Fig. 2(a) correspond to $\mathrm{Ru}$ reflections. Such twinning has been observed for other Pt-group metals deposited on sapphire as well. ${ }^{22}$ Here, the $\mathrm{Ru}$ with crystalline axes aligned with the sapphire shows a relatively higher intensity than the $30^{\circ}$ in-plane rotated lattice, indicating that the majority of the $\mathrm{Ru}$ film has lined axes with the $\mathrm{Al}_{2} \mathrm{O}_{3}$ substrate. For reference, see Fig. 2(c)) for a schematic of the $\mathrm{Ru}$ lattice aligned with the sapphire and $30^{\circ}$ in-plane rotated relative to the sapphire lattice.

To investigate the influence of annealing duration on the quality of $\mathrm{Ru}$ thin film, $30 \mathrm{~nm} \mathrm{Ru}$ films were deposited at $T_{\mathrm{s}}=700^{\circ} \mathrm{C}$, followed by in situ $700{ }^{\circ} \mathrm{C}$ anneals of varying times. Fig. 3 shows RSMs of the $\mathrm{Ru}(10 \overline{1} 3)$ reflection at $\varphi=0^{\circ}$ and $30^{\circ}$, acquired for post-anneals of 20,30 and 60 minutes. In the $\varphi=0^{\circ}$ case, the RSM was obtained by simply aligning to the nearby $\mathrm{Al}_{2} \mathrm{O}_{3}(1 \overline{2} 9)$ reflection. However, since rotating $\varphi$ by $30^{\circ}$ removes this $\mathrm{Al}_{2} \mathrm{O}_{3}$ reflection, an accurate $\mathrm{Ru}(10 \overline{1} 3)$ position was achieved in this case by alignment on the $\mathrm{Al}_{2} \mathrm{O}_{3}(10 \overline{1} 10)$ reflection, and subsequently adjusting $2 \theta$ and $\omega$. Fig. 3 illustrates the two, twinned Ru orientations: with the lattice aligned with the sapphire lattice (bottom, higher intensity), and 
$30^{\circ}$ rotated to each other (top). Increase of annealing time leads to severe reduction of one of these orientations (Fig. 3(b) and (c)): following a 60 minute post-anneal, the twinning is virtually eliminated, leaving only the film orientation aligned with the substrate, with no inplane lattice rotation: $\mathrm{Ru}(0001)[10 \overline{1} 0] \| \mathrm{Al}_{2} \mathrm{O}_{3}(0001)[10 \overline{1} 0]$. Whilst inconsistent with the lowest lattice mismatch $(-1.6 \%)$ relationship discussed above: $\mathrm{Ru}(0001)[10 \overline{1} 0] \| \mathrm{Al}_{2} \mathrm{O}_{3}(0001)[11 \overline{2} 0]^{19,20}$, this film orientation has also been reported by Sutter et al. ${ }^{16}$ for films deposited by RF sputtering at $T_{\mathrm{s}}=650{ }^{\circ} \mathrm{C}$, and post-annealed at $850{ }^{\circ} \mathrm{C}$ for 15 minutes. Such epitaxial relationship has been attributed to the accommodation of the large epitaxial mismatch $(13.6 \%)$ to relaxation of the film lattice through a network of interfacial dislocations ${ }^{19,20}$.

The out-of-plane lattice constants of our Ru films were calculated from the (0002) and (0004)

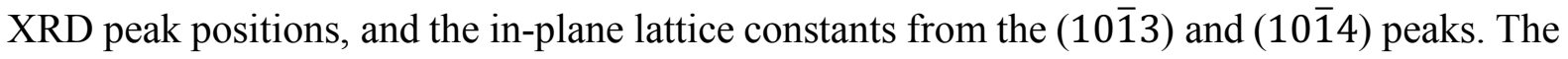
lattice parameters are comparable to the bulk values $(a=2.706 \AA, c=4.281 \AA)^{31}$, see Table 2, indicating complete strain relaxation. The out of plane lattice parameter $(c)$ is within $0.03 \%$ of the bulk and the in-plane within $0.07 \%$ of the bulk value.

Whilst we have demonstrated that growth at $700{ }^{\circ} \mathrm{C}$, followed by a 60 minute $700{ }^{\circ} \mathrm{C}$ anneal, can lead to very high quality, single-orientation $\mathrm{Ru}$ films, with bulk lattice parameters, films thinner than $30 \mathrm{~nm}$ proved to be of lower quality, due to a higher surface roughness - see Fig. 1(b). For future antiferromagnetic $\mathrm{Mn}_{3} X$ spintronic devices, a thick Ru buffer layer hinders the measurement of phenomenon like the anomalous Hall and spin Hall effects, since most of the electric current will flow through the thick Ru buffer layer due to its high conductivity. Therefore we sought to find a different deposition process to realize thinner $\mathrm{Ru}$ films that maintain a comparable crystalline quality as the $30 \mathrm{~nm}$ film prepared by route (i).

Route (ii): $2 \theta / \theta$ XRD scans of $7 \mathrm{~nm}, 15 \mathrm{~nm}$ and $30 \mathrm{~nm}$ thick Ru films, deposited by RFmagnetron sputtering at room temperature and in situ post-annealed at $700{ }^{\circ} \mathrm{C}$ for 60 minutes, are shown in Fig. 4(a). In contrast with Fig. 1(a), all XRD scans show thickness fringes. XRR scans (Fig. 4(b)) exhibit clear Kiessig fringes, indicating smooth interfaces and confirming that high quality film growth was achieved for all thicknesses. The estimated values for roughness obtained from the simulated XRR data for the $30 \mathrm{~nm}, 15 \mathrm{~nm}$ and $7 \mathrm{~nm}$ are $3.3 \AA, 2.5 \AA$ and $2.7 \AA$ respectively. Fig. 4(c) shows $\varphi$-scans from the $\mathrm{Ru}(10 \overline{1} 1)$ and $\mathrm{Al}_{2} \mathrm{O}_{3}(11 \overline{2} 3)$ reflections of the $30 \mathrm{~nm}$ film, illustrating the in-plane film orientation. In this case 6-fold symmetry is observed in the Ru film, with the film and substrate peaks aligned, suggesting the lower lattice misfit $\mathrm{Ru}(0001)[10 \overline{1} 0] \| \mathrm{Al}_{2} \mathrm{O}_{3}(0001)[11 \overline{2} 0]$ epitaxial relationship (i.e. the $\mathrm{Ru}$ lattice is $30^{\circ}$ 
rotated in-plane relative to $c-\mathrm{Al}_{2} \mathrm{O}_{3}$ ). This orientation has also been reported by Brandenburg et al. ${ }^{19}$ (using PLD) and Milosevic et al. ${ }^{20}$ (DC-magnetron sputtering), with film growth at a low $T_{\mathrm{s}}$ and a subsequent higher temperature in situ annealing.

RSMs of the $\mathrm{Ru}(10 \overline{1} 3)$ reflection at $\varphi=0^{\circ}$ and $30^{\circ}$ (Supplementary Note 3 ) for a $30 \mathrm{~nm}$ thick film support the results of Fig. 4.: no Ru (1013) reflection is observed at $\varphi=30^{\circ}$, confirming the $\mathrm{Ru}(0001)[10 \overline{1} 0] \| \mathrm{Al}_{2} \mathrm{O}_{3}(0001)[11 \overline{2} 0]$ epitaxial relationship. The lattice constants were

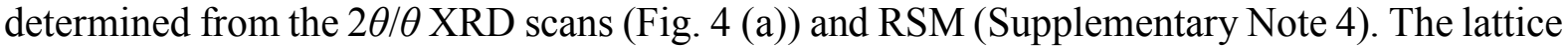
parameters, given in Table 2, are comparable to the bulk values, indicating that the films are relaxed, even at $7 \mathrm{~nm}$. The out of plane lattice constant $(c)$ is within $\sim 0.02 \%$ of the bulk and the in-plane $(a)$ varies within $\sim 0.04-0.18 \%$ of the bulk value.

The structure and orientation of a $7 \mathrm{~nm}$ thick $\mathrm{Ru}$ film, deposited at room temperature and postannealed for 60 minutes in situ at $700{ }^{\circ} \mathrm{C}$, was investigated by high resolution S/TEM (Fig. 5). Fig. 5(a) shows the $\mathrm{Ru}$ film on top of the $c-\mathrm{Al}_{2} \mathrm{O}_{3}$ substrate. The film was observed to have a uniform thickness (confirmed over a $1.5 \mu \mathrm{m}$ length scale) and low interfacial roughness, with $\mathrm{Ru}(0001)$ lattice planes observed to be parallel to the substrate surface. The image also shows a very smooth $\mathrm{Al}_{2} \mathrm{O}_{3} / \mathrm{Ru}$ interface with only three, monolayer high steps across a range of $13 \mathrm{~nm}$ width. The inset in Fig. 5(a) shows an enlarged area, confirming very high crystalline film quality. SAED patterns, with the electron beam parallel to the $c-\mathrm{Al}_{2} \mathrm{O}_{3}[11 \overline{2} 0]$ zone axis,

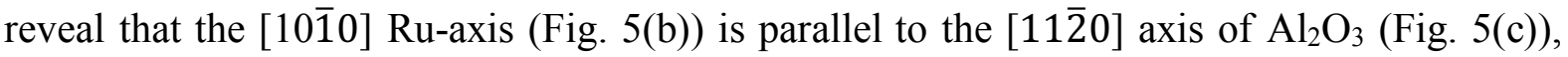
confirming the epitaxial relationship inferred from the XRD data.

The results from route (i) imply that the higher deposition temperature promotes mobility of $\mathrm{Ru}$ adatoms and clusters on the substrate surface during film growth, leading to island-growth. As the film thickens, individual islands coalesce into larger grains, and twin boundaries form between islands that have nucleated in the two different symmetry-related orientations: high misfit (aligned with the substrate crystal axes), and low misfit (with a $30^{\circ}$ rotation). Fig. 2(b) illustrates that the higher misfit (13.6\%) orientation is dominant with a more intense Ru (1011) reflection. A longer post-deposition anneal promotes domain growth (loss of twin boundaries), with this dominant orientation increasing and, after 60 minutes, this single film orientation remains. It appears that the high lattice mismatch is accommodated through relaxation during annealing, leaving a network of interfacial dislocations ${ }^{16}$. In contrast, deposition at room temperature (route (ii)) is likely to take place through layer-by-layer growth, leading to the lower misfit $\left(30^{\circ}\right.$ rotated) crystalline orientation, smaller grain sizes (see calculated lateral coherence lengths in Supplementary Note 2) and smooth surfaces for all film thicknesses. 
Finally, $30 \mathrm{~nm}$ thick $\mathrm{Mn}_{3} \mathrm{Ga}$ and $\mathrm{Mn}_{3} \mathrm{Sn}$ films were then deposited on an optimized $7 \mathrm{~nm} \mathrm{Ru}$ buffer layer (route (ii)) to verify if epitaxial and homogenous $\mathrm{Mn}_{3} X$ films can be achieved. Fig. 6 summarizes the $\mathrm{Mn}_{3} \mathrm{Ga}$ and $\mathrm{Mn}_{3} \mathrm{Sn}$ structural characterization using XRD. Fig.6(a) and (b) shows XRD $2 \theta / \theta$ scans around the (0002) $\mathrm{Mn}_{3} \mathrm{Ga}$ and $\mathrm{Mn}_{3} \mathrm{Sn}$ peaks respectively (no other phases, nor orientations, were observed), indicating that highly epitaxial growth of the films on an optimized $\mathrm{Ru}$ buffer layer has been achieved. Both films are relaxed as the $\mathrm{Mn}_{3} X$ reflection do not align in $S_{\mathrm{x}}$ with the $\mathrm{Al}_{2} \mathrm{O}_{3}(11 \overline{2} 9)$ reflections as indicated by the RSMs, in Fig. 6(c) and (d) for $\mathrm{Mn}_{3} \mathrm{Ga}$ and $\mathrm{Mn}_{3} \mathrm{Sn}$ respectively. The epitaxial relationship as implied by Fig. 6(c) and (d) for $\mathrm{Mn}_{3} \mathrm{Ga}$ and $\mathrm{Mn}_{3} \mathrm{Sn}$ and as supported by $\varphi$-scans (Supplementary Note 5) is: $\mathrm{Mn}_{3} X(X=\mathrm{Ga}, \mathrm{Sn})(0001)[20 \overline{2} 0]\|\mathrm{Ru}(0001)[10 \overline{1} 0]\| \mathrm{Al}_{2} \mathrm{O}_{3}(0001)[11 \overline{2} 0]$. The highly epitaxial and single-phase $\mathrm{Mn}_{3} \mathrm{Ga}$ and $\mathrm{Mn}_{3} \mathrm{Sn}$ thin films deposited on the on the optimized thin $\mathrm{Ru}$ buffer layer have bulk-like lattice constants (Table 3) and are therefore promising to investigate the intriguing spintronic effects originating from the non-collinear antiferromagnetic order of these systems.

In summary, we have presented two deposition routes for $\mathrm{Ru}$ thin films on $c-\mathrm{Al}_{2} \mathrm{O}_{3}$ substrates. Depositing the films at $700{ }^{\circ} \mathrm{C}$ resulted in twinned $\mathrm{Ru}$ orientations and rough surfaces when the nominal film thickness was $\leq 15 \mathrm{~nm}$. Subsequent in-situ post annealing at $700{ }^{\circ} \mathrm{C}$ led to a single in-plane orientation of $\mathrm{Ru}\left(\mathrm{Ru} 0^{\circ}\right.$ rotated relative to the $c-\mathrm{Al}_{2} \mathrm{O}_{3}$ substrate), however did not improve the surface morphology. Depositing at room temperature followed by in-situ postannealing at $700{ }^{\circ} \mathrm{C}$ resulted in the higher quality films down to $7 \mathrm{~nm}$ with no twinned grains. In addition, we also showed that highly epitaxial and single phase $\mathrm{Mn}_{3} X(X=\mathrm{Ga}, \mathrm{Sn})$ on top of the optimized $\mathrm{Ru}$ film can be grown with a $\operatorname{Mn}_{3} X(X=\mathrm{Ga}$, Sn) (0001)[20 $\overline{2} 0]\|\mathrm{Ru}(0001)[10 \overline{1} 0]\| \mathrm{Al}_{2} \mathrm{O}_{3}(0001)[11 \overline{2} 0]$ epitaxial relationship, that promises bulk-like magnetic and transport properties, which will be investigated in future studies.

\section{Supplementary}

See supplementary material for the following notes:

Note 1: Atomic Force Microscopy

Note 2: Vertical and lateral coherence length for the $\mathrm{Ru}$ and the $\mathrm{Mn}_{3} X$ films.

Note 3: RSM of the $\mathrm{Ru}(10 \overline{1} 3)$ reflection for a $30 \mathrm{~nm}$ thick $\mathrm{Ru}$ film taken at $\varphi=0^{\circ}$ and $30^{\circ}$. Note 4: RSM of the $\mathrm{Ru}(10 \overline{1} 3)$ reflection for $30 \mathrm{~nm}, 15 \mathrm{~nm}$, and $7 \mathrm{~nm}$ thick Ru films.

Note 5: $\varphi$-scans of the $\mathrm{Mn}_{3} X(X=\mathrm{Ga}, \mathrm{Sn})(20 \overline{2} 1), \mathrm{Ru}(10 \overline{1} 1)$ and $\mathrm{Al}_{2} \mathrm{O}_{3}(11 \overline{2} 3)$ reflections. 


\section{Acknowledgements}

This work was funded by the H2020-MSCA-ITN-2014 SELECTA (grant agreement no. 642642 of the European Commission). 


\section{Tables}

Table 1. The lattice constants for $30 \mathrm{~nm}$ thick Ru films deposited at $700{ }^{\circ} \mathrm{C}$ and post-annealed in situ for 20,30 and 60 minutes.

\begin{tabular}{|c|c|c|c|}
\hline & $\boldsymbol{c}(\boldsymbol{\AA})$ & \multicolumn{2}{|c|}{$\boldsymbol{a ( \AA )}$} \\
\hline $\begin{array}{c}\text { Post Anneal Time } \\
(\mathbf{m i n})\end{array}$ & & $\boldsymbol{\varphi}=\mathbf{0}^{\circ}$ & $\boldsymbol{\varphi}=\mathbf{3 0}^{\circ}$ \\
\hline 20 & $4.282 \pm 0.001$ & $2.707 \pm 0.002$ & $2.706 \pm 0.001$ \\
\hline 30 & $4.282 \pm 0.001$ & $2.708 \pm 0.002$ & $2.707 \pm 0.001$ \\
\hline 60 & $4.282 \pm 0.001$ & $2.708 \pm 0.001$ & $2.707 \pm 0.001$ \\
\hline
\end{tabular}

Table 2. Lattice constants for $7 \mathrm{~nm}, 15 \mathrm{~nm}$, and $30 \mathrm{~nm} \mathrm{Ru}$ films deposited at room temperature and post-annealed in situ at $700{ }^{\circ} \mathrm{C}$ for 60 minutes.

\begin{tabular}{|c|c|c|}
\hline Thickness (nm) & $\boldsymbol{c}(\boldsymbol{\AA})$ & $\boldsymbol{a}(\boldsymbol{\AA})$ \\
\hline 7 & $4.282 \pm 0.001$ & $2.711 \pm 0.002$ \\
\hline 15 & $4.281 \pm 0.001$ & $2.707 \pm 0.001$ \\
\hline 30 & $4.280 \pm 0.001$ & $2.708 \pm 0.001$ \\
\hline
\end{tabular}

Table 3. Lattice constants for $30 \mathrm{~nm} \mathrm{Mn}{ }_{3} \mathrm{Ga}$ and $\mathrm{Mn}_{3} \mathrm{Sn}$ films deposited on a $7 \mathrm{~nm}$ Ru buffer (route (ii)).

\begin{tabular}{|c|c|c|}
\hline Films & $\boldsymbol{c}(\boldsymbol{\AA})$ & $\boldsymbol{a}(\boldsymbol{\AA})$ \\
\hline $\mathrm{Mn}_{3} \mathrm{Ga}$ & $4.353 \pm 0.002$ & $5.433 \pm 0.002$ \\
\hline $\mathrm{Mn}_{3} \mathrm{Sn}$ & $4.530 \pm 0.001$ & $5.670 \pm 0.002$ \\
\hline
\end{tabular}




\section{Figures}
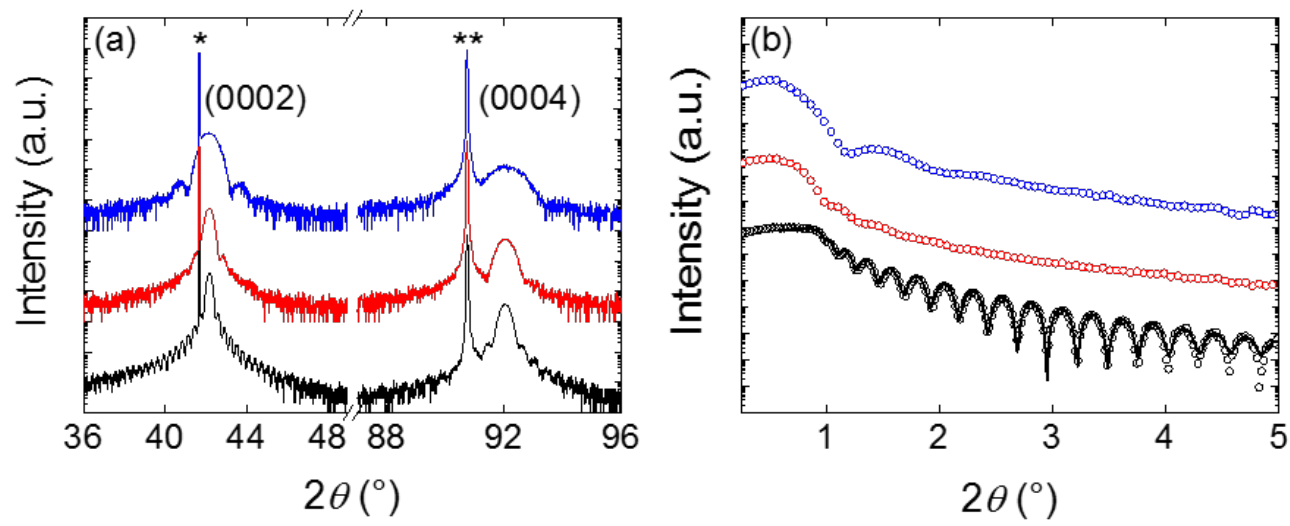

Figure 1. (a) $2 \theta / \theta \mathrm{XRD}$, and (b) XRR scans for $30 \mathrm{~nm}$ (black), $15 \mathrm{~nm}$ (red) and $5 \mathrm{~nm}$ (blue) $\mathrm{Ru}$ films deposited at $700{ }^{\circ} \mathrm{C}$ and post-annealed in situ for 20 minutes at the same temperature. $*$ and ** indicate the $\mathrm{Al}_{2} \mathrm{O}_{3}(0006)$ and (000 12) reflections respectively. The open circles and the solid lines represent the measured and simulated curves respectively.

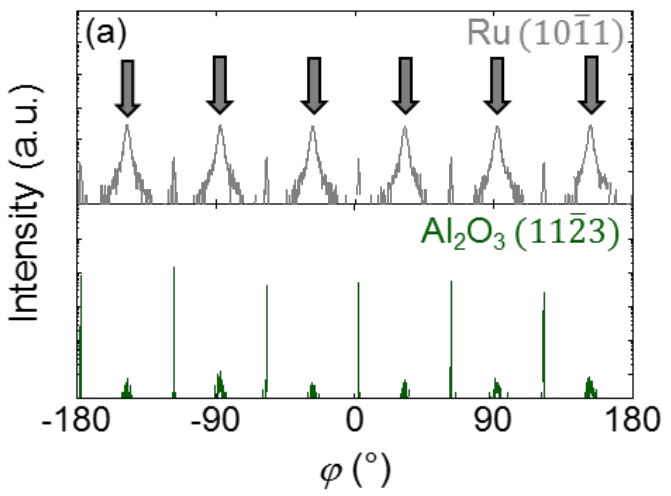

(c) Unrotated Ru lattice

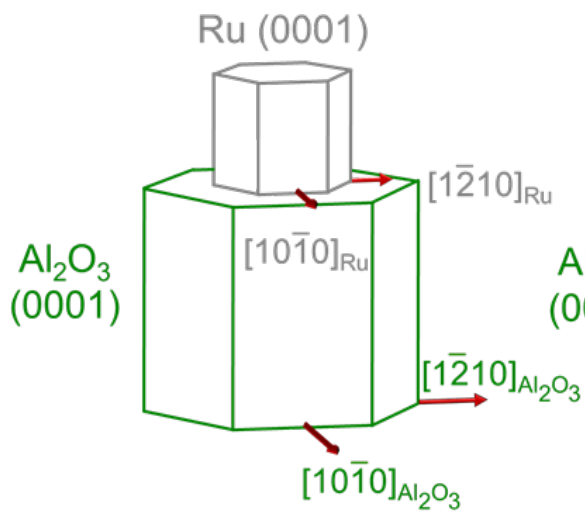

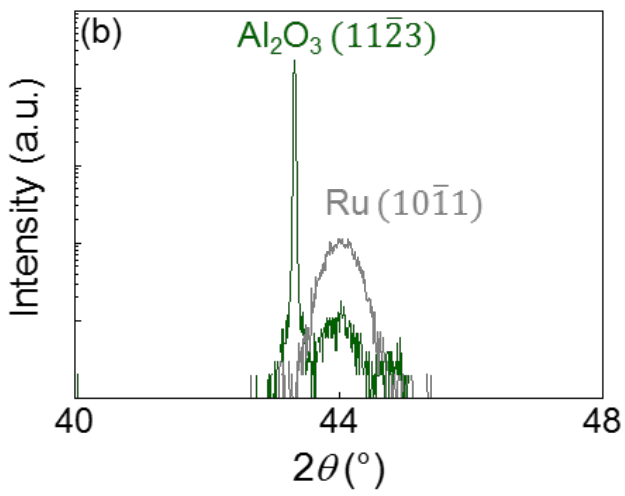

$30^{\circ}$ rotated $\mathrm{Ru}$ lattice

Figure 2. (a) $\mathrm{Al}_{2} \mathrm{O}_{3}(11 \overline{2} 3)$ (green) and $\mathrm{Ru}(10 \overline{1} 1$ ) (grey) $\varphi$-scans for a $30 \mathrm{~nm}$ thick $\mathrm{Ru}$ film sample deposited at $700{ }^{\circ} \mathrm{C}$ and post-annealed (20 minutes) at the same temperature. (b) $2 \theta / \theta$ XRD scans at $\chi=61.20^{\circ}$ with $\varphi=0^{\circ}$ (green) and $\varphi=30^{\circ}$ (grey). (c) Schematic of an Ru lattice aligned with the sapphire lattice (left) and $30^{\circ}$ rotated about the sapphire lattice. 


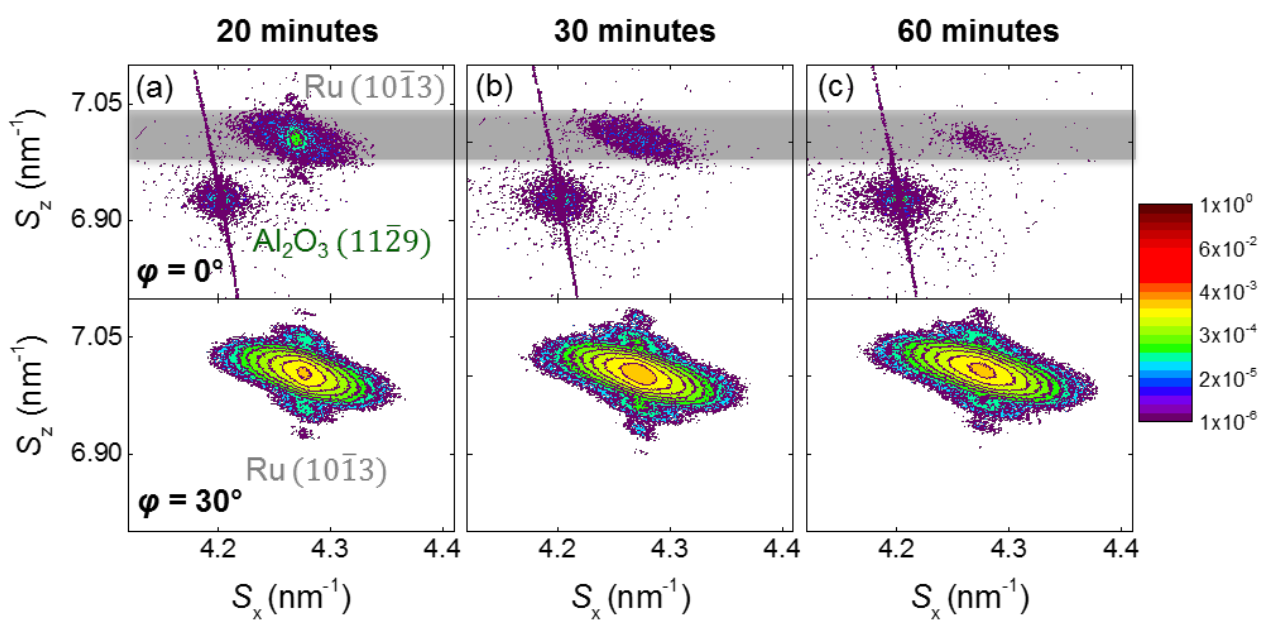

Figure 3. $\mathrm{Ru}(10 \overline{1} 3) \mathrm{RSMs}$ at $\varphi=0^{\circ}$ and $\varphi=30^{\circ}$ for $30 \mathrm{~nm}$ thick Ru films deposited at $700{ }^{\circ} \mathrm{C}$ and post-annealed for 20 minutes (a), 30 minutes (b) and 60 minutes (c). The grey shading represents the position of the $\mathrm{Ru}(10 \overline{1} 3)$ reflection in the case of a $30^{\circ}$-rotated $\mathrm{Ru}$ lattice relative to $\mathrm{Al}_{2} \mathrm{O}_{3}$.
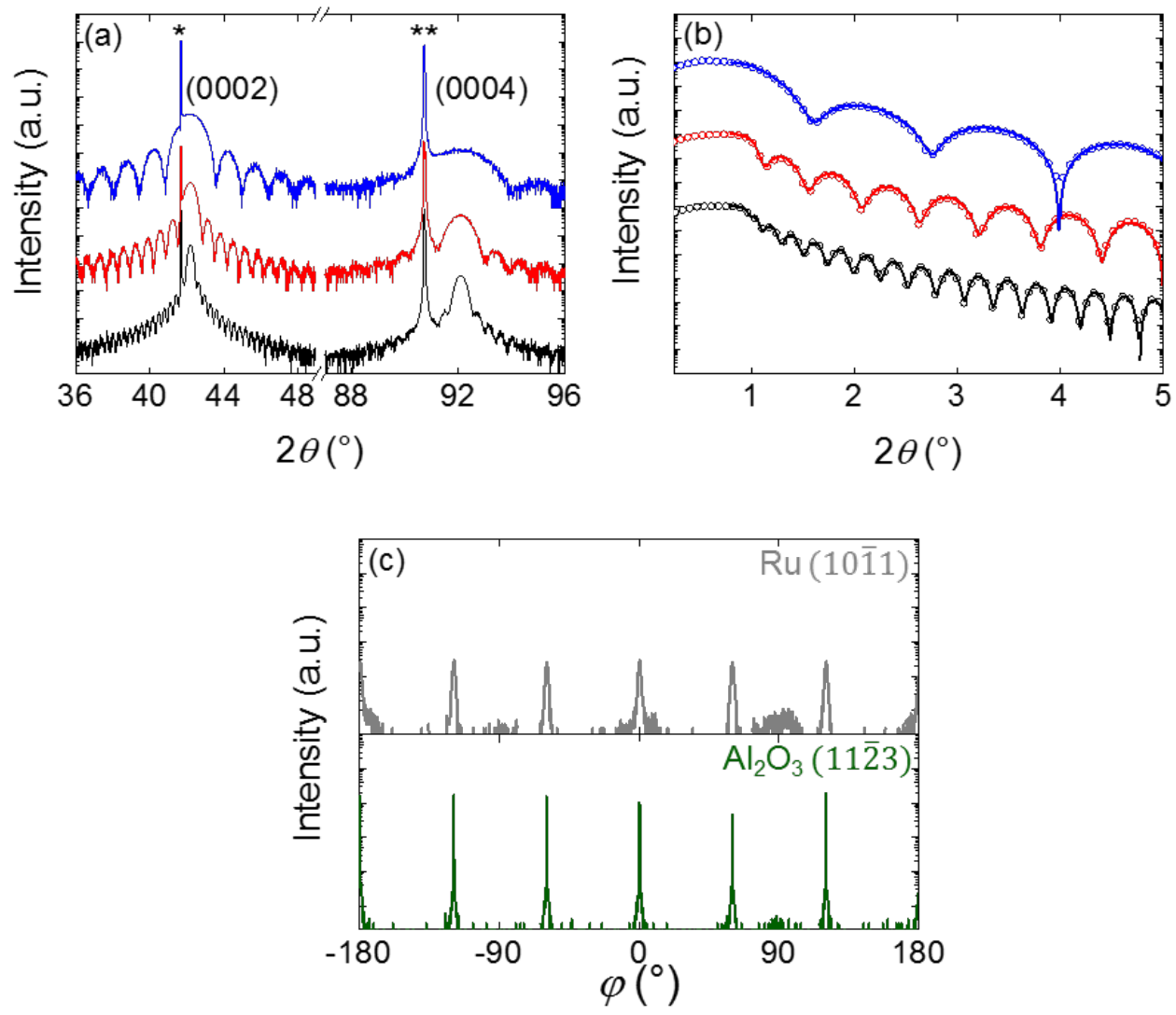

Figure 4. (a) $2 \theta / \theta$ XRD scans and (b) XRR scans for $30 \mathrm{~nm}$ (black), $15 \mathrm{~nm}$ (red), and $7 \mathrm{~nm}$ (blue) $\mathrm{Ru}$ films deposited at room temperature and post-annealed in situ at $700{ }^{\circ} \mathrm{C}$ for 60 minutes. The open circles and the solid lines represent the measured and simulated curves respectively. (c) $\mathrm{Al}_{2} \mathrm{O}_{3}(11 \overline{2} 3)$ (green) and $\mathrm{Ru}(10 \overline{1} 1)$ (grey) $\varphi$-scans for a $30 \mathrm{~nm}$ thick Ru film deposited at room temperature and post-annealed in situ for 60 minutes at $700{ }^{\circ} \mathrm{C}$. ${ }^{*}$ and ** indicate the $\mathrm{Al}_{2} \mathrm{O}_{3}(0006)$ and $(000$ 12) reflections respectively. 

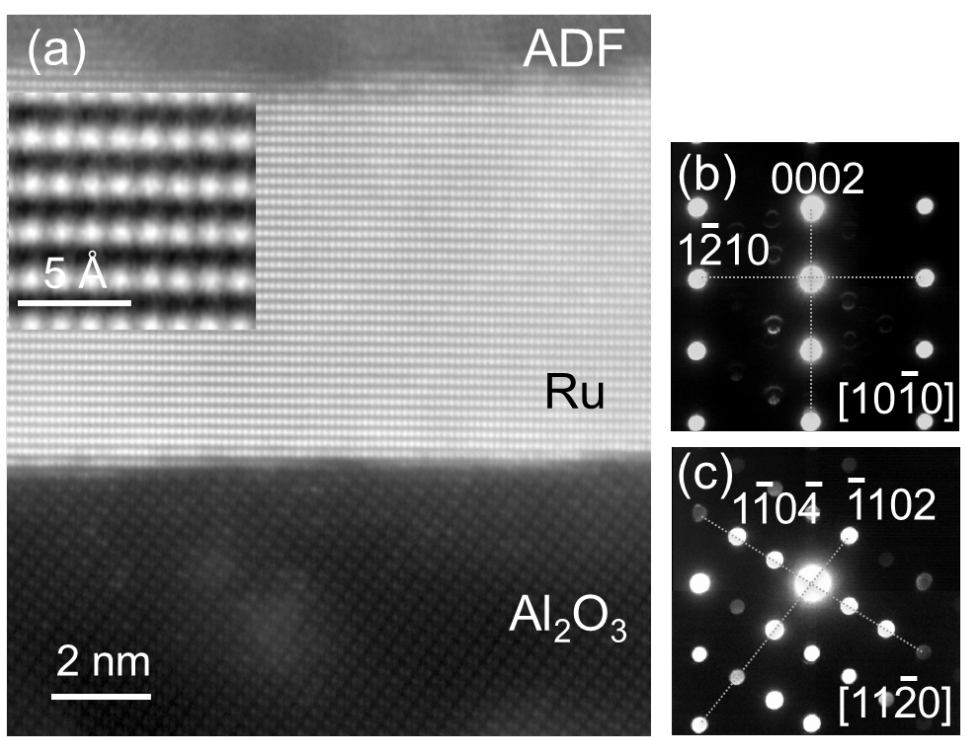

Figure 5. (a) A STEM cross-sectional image of a $7 \mathrm{~nm}$ thick $\mathrm{Ru}$ film deposited on $c-\mathrm{Al}_{2} \mathrm{O}_{3}$ via route (ii). The inset shows a HAADF-STEM image of enlarged area of the Ru film. (b) SAED patterns obtained along the [1120] zone axis of $\mathrm{Al}_{2} \mathrm{O}_{3}$ for the (b) $\mathrm{Ru}$ and (c) $\mathrm{Al}_{2} \mathrm{O}_{3}$ layers. The dashed grey lines are guides for the eye only.
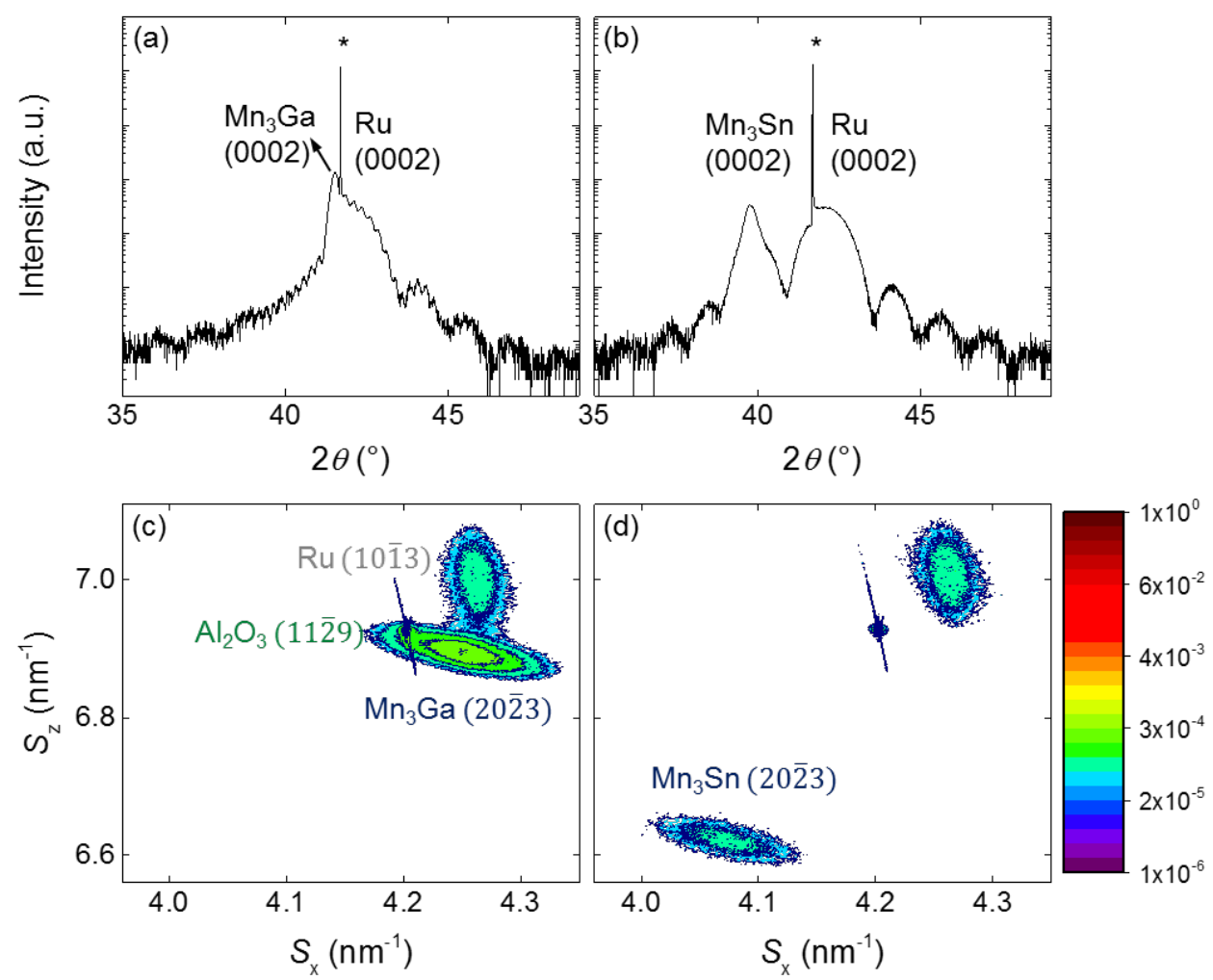

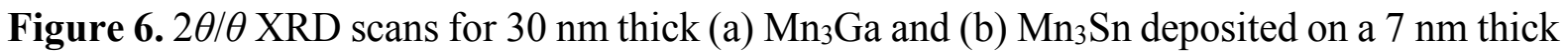
Ru film deposited at $700{ }^{\circ} \mathrm{C}$ and post-annealed in situ for 20 minutes at the same temperature.

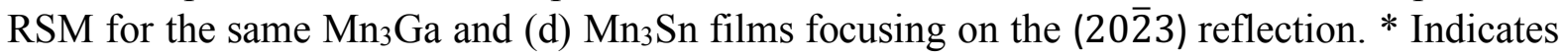
the $\mathrm{Al}_{2} \mathrm{O}_{3}$ (0006) reflection. 


\section{References}

${ }^{1}$ T. Jungwirth, X. Marti, P. Wadley, and J. Wunderlich, Nat. Nanotechnol. 11, 231 (2016).

${ }^{2}$ K. Kuroda, T. Tomita, M.-T. Suzuki, C. Bareille, A.A. Nugroho, P. Goswami, M. Ochi, M. Ikhlas, M. Nakayama, S. Akebi, R. Noguchi, R. Ishii, N. Inami, K. Ono, H. Kumigashira, A. Varykhalov, T. Muro, T. Koretsune, R. Arita, S. Shin, T. Kondo, and S. Nakatsuji, Nat. Mater. 16, 1090 (2017).

${ }^{3}$ S. Nakatsuji, N. Kiyohara, and T. Higo, Nature 527, 212 (2015).

${ }^{4}$ M. Ikhlas, T. Tomita, T. Koretsune, M.T. Suzuki, D. Nishio-Hamane, R. Arita, Y. Otani, and S. Nakatsuji, Nat. Phys. 13, 1085 (2017).

${ }^{5}$ T. Higo, H. Man, D.B. Gopman, L. Wu, T. Koretsune, O.M.J. Van 'T Erve, Y.P. Kabanov, D. Rees, Y. Li, M.T. Suzuki, S. Patankar, M. Ikhlas, C.L. Chien, R. Arita, R.D. Shull, J. Orenstein, and S. Nakatsuji, Nat. Photonics 12, 73 (2018).

${ }^{6}$ A.K. Nayak, J.E. Fischer, Y. Sun, B. Yan, J. Karel, A.C. Komarek, C. Shekhar, N. Kumar, W. Schnelle, J. Kübler, C. Felser, and S.S.P. Parkin, Sci. Adv. 2, e1501870 (2016).

${ }^{7}$ H. Kurt, K. Rode, M. Venkatesan, P. Stamenov, and J.M.D. Coey, Phys. Status Solidi Basic Res. 248, 2338 (2011).

${ }^{8}$ H. Wu, I. Sudoh, R. Xu, W. Si, C.A.F. Vaz, J.Y. Kim, G. Vallejo-Fernandez, and A. Hirohata, J. Phys. D. Appl. Phys. 51, 215003 (2018).

${ }^{9}$ A. Markou, J.M. Taylor, A. Kalache, P. Werner, S.S.P. Parkin, and C. Felser, Phys. Rev. Mater. 2, 051001 (2018).

${ }^{10}$ T. Ogasawara, J. Kim, Y. Ando, and A. Hirohata, J. Magn. Magn. Mater. 473, 7 (2019).

${ }^{11}$ Y. You, X. Chen, X. Zhou, Y. Gu, R. Zhang, F. Pan, and C. Song, Adv. Electron. Mater. 5, 1800818 (2019).

${ }^{12}$ H. Reichlova, T. Janda, J. Godinho, A. Markou, D. Kriegner, R. Schlitz, J. Zelezny, Z. Soban, M. Bejarano, H. Schultheiss, P. Nemec, T. Jungwirth, C. Felser, J. Wunderlich, and S.T.B. Goennenwein, Nat. Commun. 10, 5459 (2019).

${ }^{13}$ W. Hsu, H. Kao, and Z. Lin, J. Cryst. Growth 436, 46 (2016).

${ }^{14}$ J.L. Schroeder, A.S. Ingason, J. Rosén, and J. Birch, J. Cryst. Growth 420, 22 (2015).

${ }^{15}$ R. Suzuki, A. Kawaharazuka, and Y. Horikoshi, J. Cryst. Growth 311, 2021 (2009).

${ }^{16}$ P.W. Sutter, P.M. Albrecht, and E.A. Sutter, Appl. Phys. Lett. 97, 213101 (2010).

${ }^{17}$ S. Yoshii, K. Nozawa, K. Toyoda, N. Matsukawa, A. Odagawa, and A. Tsujimura, Nano Lett. 11, 2628 (2011).

${ }^{18}$ S. Yamada, Y. Nishibe, M. Saizaki, H. Kitajima, S. Ohtsubo, A. Morimoto, T. Shimizu, K. Ishida, and Y. Masaki, Jpn. J. Appl. Phys. 41, L206 (2002).

${ }^{19}$ J. Brandenburg, R. Hühne, L. Schultz, and V. Neu, Phys. Rev. B 79, 054429 (2009).

${ }^{20}$ E. Milosevic, S. Kerdsongpanya, A. Zangiabadi, K. Barmak, K.R. Coffey, and D. Gall, J. Appl. Phys. 124, 165105 (2018). 
${ }^{21}$ T. Kim, T. Kawae, N. Ikegami, S. Yamada, Y. Yonezawa, K. Takahashi, A. Morimoto, and M. Kumeda, Jpn. J. Appl. Phys. 47, 6374 (2008).

${ }^{22}$ S. Gsell, M. Fischer, M. Schreck, and B. Stritzker, J. Cryst. Growth 311, 3731 (2009).

${ }^{23}$ T. Shibutami, K. Kawano, N. Oshima, S. Yokoyama, and H. Funakubo, Electrochem. Solid-State Lett. 6, C117 (2003).

${ }^{24}$ K. Kawano, A. Nagai, H. Kosuge, T. Shibutami, N. Oshima, and H. Funakubo, Electrochem. Solid-State Lett. 9, C107 (2006).

${ }^{25}$ M. Change, S. Ganguli, and N. Maity, US 7,264,846 B2 (2007).

${ }^{26}$ T.N. Arunagiri, Y. Zhang, O. Chyan, M. El-Bouanani, M.J. Kim, K.H. Chen, C.T. Wu, and L.C. Chen, Appl. Phys. Lett. 86, 083104 (2005).

${ }^{27}$ S.H. Park, S.O. Kim, T.D. Lee, H.S. Oh, Y.S. Kim, N.Y. Park, and D.H. Hong, J. Appl. Phys. 99, 08E701 (2006).

${ }^{28}$ E. Krén and G. Kádár, Solid State Commun. 8, 1653 (1970).

${ }^{29}$ E. Krén, J. Paitz, G. Zimmer, and E. Zsoldos, Physica 80B, 226 (1975).

${ }^{30}$ P. Scherrer, Nachr Ges Wiss Göttingen 26, 387 (1912).

${ }^{31}$ M. Černohorský, Acta Crystallogr. 13, 823 (1960).

${ }^{32}$ B.D. Cullity and S.R. Stock, Elements of X-Ray Diffraction, 3rd ed. (Pearson Education Limited, London, UK, 2014). 